\title{
Hierarchical-temporal Data Visualization Using a Tree-ring Metaphor ${ }^{\star}$
}

\author{
Roberto Therón \\ Departamento de Informática y Automática, \\ Universidad de Salamanca, Salamanca, 37008, Spain \\ theron@usal.es
}

\begin{abstract}
This paper describes a novel and efficient visualization technique intended for hierarchical-temporal data using a tree-ring like layout. Temporal hierarchies appear in numerous fields such as genealogy, evolution taxonomies or time lines. In many cases, state-of-the-art static diagrams are produced in these fields. By using several information visualization strategies, such as focus + context, the tree-ring approach has the ability to visualize and navigate these, potentially complex, hierarchies trough time. Thus, a deeper insight into the problem at hand can be gained.
\end{abstract}

Key words: tree-ring layout, information visualization, focus + context

\section{Introduction}

In the last few years the visualization of hierarchical data has been a subject of great interest in information visualization research. Hierarchies are often represented as trees, i.e., a special kind of graph. Graphs are the fundamental structural representation of structured data [1], so the hierarchy visualization problem becomes a part of the graph visualization problem.

Hierarchies are used in many areas of application, including computer file systems, taxonomies, genealogy, phylogenesis, evolutionary trees, etc. The information visualization community has explored many different approaches, focusing on navigation and interaction, that help to overcome some of the limitations present in graph drawing. For the latter, the book of Battista et al.[2] covers all the key aspects, while Herman et al. provide a thorough survey of information visualization applied to graph visualization and interaction[1].

Although the visualization of hierarchies has been extensively studied in the literature, very few works related to the visualization and exploration of hierarchical data sets that represent a temporal evolution have been done. Furthermore, in many situations visualizations should emphasize temporal relationships

\footnotetext{
* This work was supported by the MCyT of Spain under Integrated Action (SpainFrance) HF2004-0277 and by the Junta de Castilla y León under project SA042/02. The author would like to acknowledge Antonio Hernández Serrano for his assistance in the implementation of this work.
} 
and patterns. This paper presents Treevolution, a novel visualization tool that uses a tree-ring metaphor in combination with focus + context techniques, that can be useful for hierarchical-temporal data visualization.

The rest of this paper is organized as follows: next, a review of works related to hierarchical data and time visualization is presented. In Section 2 the treering metaphor is explained. The third section is devoted to a case study: the visualization of the computer language history using Treevolution. To finalize, the main conclusions and future work are described.

\subsection{Related work}

Many display layout techniques have been developed for the visualization of hierarchical data sets like Cone tree, Treemap[3], Hyperbolic tree[4], etc. Several works have dealt with focus + context techniques for visualizing and manipulating large hierarchies. Yee et al. [5] use the well known radial tree layout method[6][2], in which the focused node is placed in center of display, and all other nodes are rendered on appropriate circular level around that selected focused node. Also this approach makes use of different animation techniques for supporting interactive exploration of the graph. In [7] a software framework for creating dynamic visualizations of both structured and unstructured data was developed.

An important aspect is how the process of determining the position and size of each visual object that is displayed in a presentation can automated. Effective layout is one of the most important aspects of creating an information presentation, because that presentation it is intended to be viewed and manipulated by people. A survey of automated layout techniques for information presentations can be found in $[8]$.

In [9] focus + context techniques were used to compare the structure of large phylogenetic trees. Munzner et al. proposed a new rectilinear focus + context technique for navigation that is well suited to the dynamic linking of side-by-side views while guaranteeing landmark visibility and constant frame rates.

Recently, Morris et al.[10] have dealt with the visualization of temporal hierarchies. In their work, documents from a research front are plotted by time along a horizontal track in the time line, with related research fronts being plotted in nearby tracks according to the hierarchical structure produced during clustering.

In [11] tree-ring metaphors are introduced to enhance information content of focus areas in large time-line visualizations of objects that split or merge. The tree-ring representation shows the relative timing of splits and merges of each object. In the tree-ring metaphor for splits and merges, radial distance indicates time with the birth at the center. At a given radial distance, pieces of arc represent descendant nodes in existence at that time. A node that neither splits nor merges is represented by a circle whose radius indicates the lifetime of the node.

Finally, we would like to point out that Treevolution was developed taking into account the Computational Information Design (CID) process proposed by Benjamin Fry [12]. CID brings together design, information, and computation 
with a focus on how they support one another as parts of a combined methodology for the exploration, analysis, and representation of complex data, and processing, his Java-based software development environment aimed to simplify the construction of graphically-oriented software.

\section{The tree-ring metaphor}

As stated above, Treevolution uses a tree-ring metaphor for the temporal hierarchy layout. The following definitions will be useful for the rest of the paper ${ }^{1}$ :

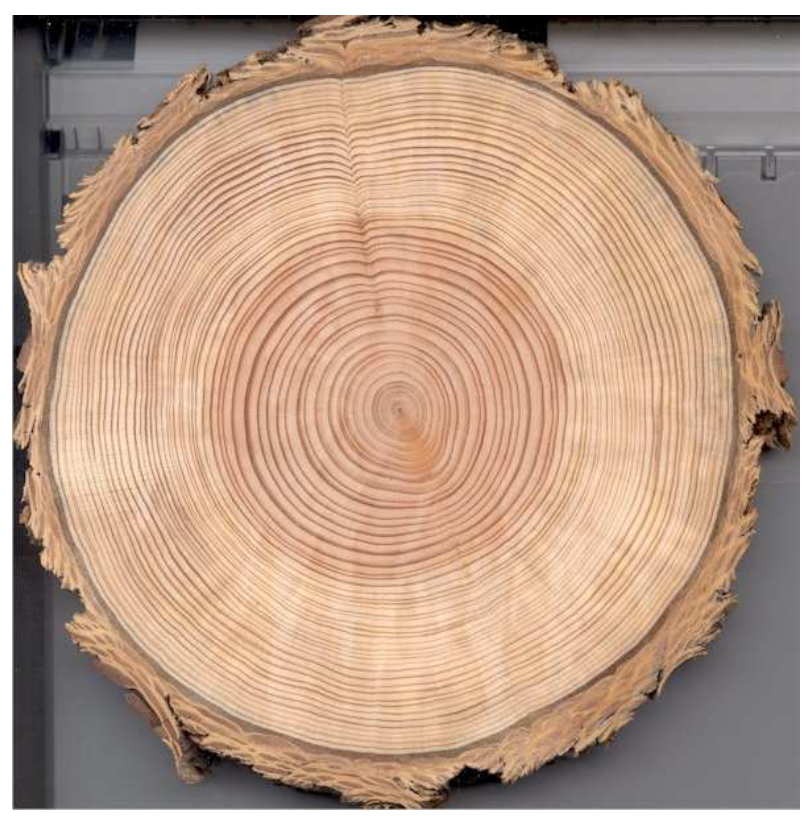

Fig. 1. Cross section of a Pseudotsuga menziesii showing almost perfect tree-rings

- Dendrochronology can be defined as The science that uses tree rings dated to their exact year of formation to analyze temporal and spatial patterns of processes in the physical and cultural sciences.

- tree ring: A layer of wood cells produced by a tree or shrub in one year, usually consisting of thin-walled cells formed early in the growing season (called earlywood) and thicker-walled cells produced later in the growing

\footnotetext{
1 The source of these elementary definitions, as well as figure 1 , is the comprehensive collection of information related to tree-rings available in Henri D. Grissino-Mayer's Ultimate Tree-Ring Web Pages, http://web.utk.edu/ grissino/
} 
season (called latewood). The beginning of earlywood formation and the end of the latewood formation form one annual ring, which usually extends around the entire circumference of the tree.

- tree-ring chronology: A series of measured tree-ring properties, such as tree-ring width or maximum latewood density, that has been converted to dimensionless indices through the process of standardization. A tree-ring chronology therefore represents departures of growth for any one year compared to average growth. For example, an index of 0.75 (or 75) for a given year indicates growth below normal (indicated by 1.00, or 100).

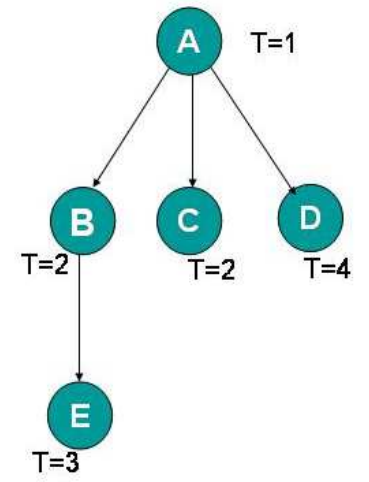

a) Temporal hierarchy

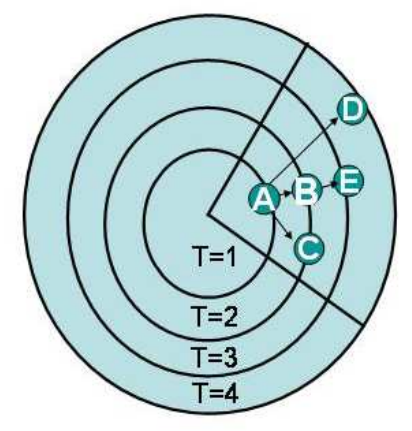

a) Tree-ring layout

Fig. 2. The tree-ring metaphor

Hence, the idea behind the tree-ring metaphor is to provide a way to visualize both timing and structure in a single diagram. In Figure 1, a cross section of a tree can be seen. In it, each tree-ring shows the annual growth; the with of the ring depends on how productive this year has been for the tree. In order to convey hierarchical information, while keeping the time information, a modification of the radial layout [6] can be used: instead of placing nodes on concentric circles according to their depth in the tree, they are placed according to its temporal information.

Figure 2 shows a simple hierarchy with temporal information. In the tree-ring layout, node $\mathrm{E}$ is placed in the fourth circle because its time is $t=4$; in this case the fact that it is a direct descendant of the root node $(\mathrm{A})$ is not important for 
node placement. The hierarchy information is kept in the directed arc (from A to E), though.

Also note that the hierarchy has been drawn occupying a circular sector. The free space could be used, in the case of having a more complex hierarchy, by placing each subtree or family in an individual sector. This concept can be seen in Figure 3. The picture shows how Treevolution, by using the tree-ring layout, visualizes several subtrees of a complex temporal hierarchy.

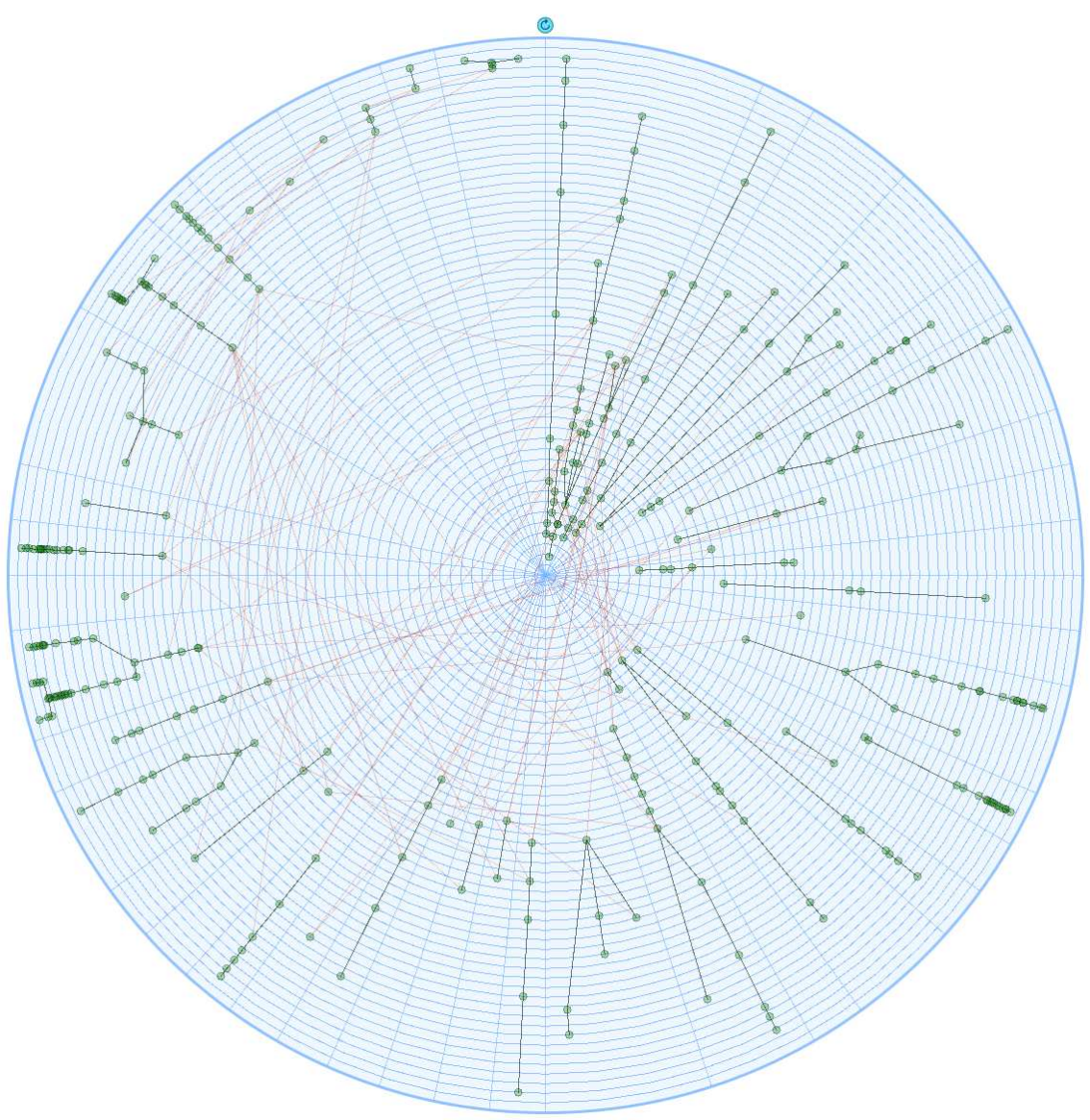

Fig. 3. Default layout of a temporal hierarchy in Treevolution

In the following sections we will see how this simple idea can be further exploited by using different information visualization techniques. 


\section{Case study: browsing the history of computer languages with Treevolution}

In order to describe the benefits of the Treevolution technique, a computer languages history diagram ${ }^{2}$ will be studied.

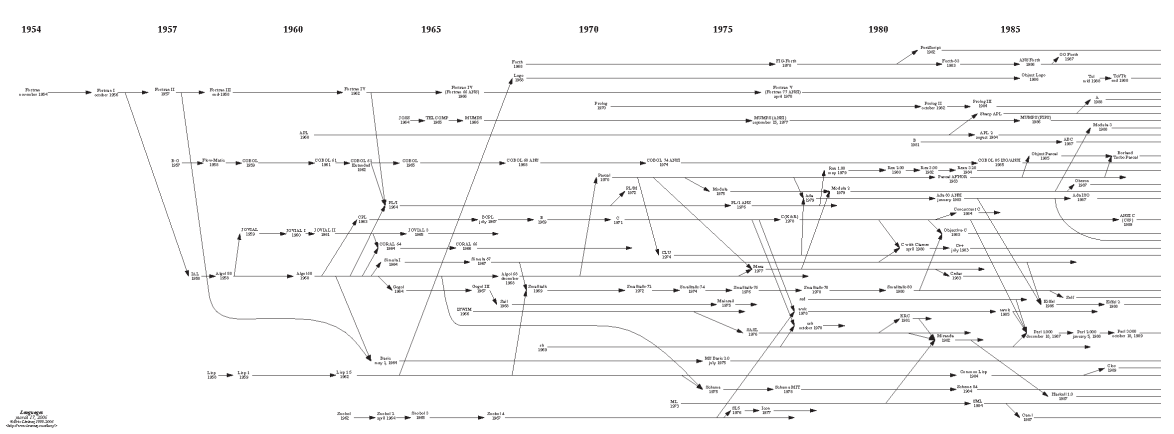

Fig. 4. Partial diagram (1957-1989) of computer languages history

Figure 4 shows the evolution of computer languages from 1957, with the advent of FORTRAN, to the current releases of popular languages such as Java or C\#. Actually, the picture only shows a small part of the history, from 1957 to 1989 , because the diagram is intended to be either horizontally scrolled with an Internet browser or to be exhibited on a wall. For our purposes, this piece of history is enough to understand that each family/tree runs from left to right, as times does. With this diagram it is very difficult to form a mental map of the evolution, since all connections between fathers and sons are mixed.

At this point, it is important to understand that the presence of a temporal component changes the types of analysis that may be done [13]. Traditional approaches for the representation of hierarchical data are not valid for faithfully preserve the information and knowledge content of the data, and prevent the viewer from understanding complex hierarchical-temporal relationships.

A user analyzing the complexity of the history of computer languages would need to answer questions like the following: How many languages have been developed? How many different families are there? Which one is the most fruitful? Is there a particular time where the evolution was faster? How many ancestors does one particular language have? How many descendants? Which is the language that had the biggest number of descendants in the smallest period of time?

Some of these questions can be answered after a tedious and meticulous analysis of the Figure 4 diagram. However, it is impossible to have an overview

\footnotetext{
${ }^{2}$ Diagram produced by Eric Levenez, http://www.levenez.com
} 
of the full history. The analyst would need to start from left to right, take some notes, and go on scrolling toward the present time. This means that important questions cannot be visually answered.

With Treevoltion the whole history (1957-2006) can be seen in a single and compact diagram. Actually, Figure 3 showed the history of computer languages in Treevolution. The same hierarchy, but this time all nodes are labeled, is shown in figure 5 . Note that light red edges depict the ancestors of nodes pertaining to other families.

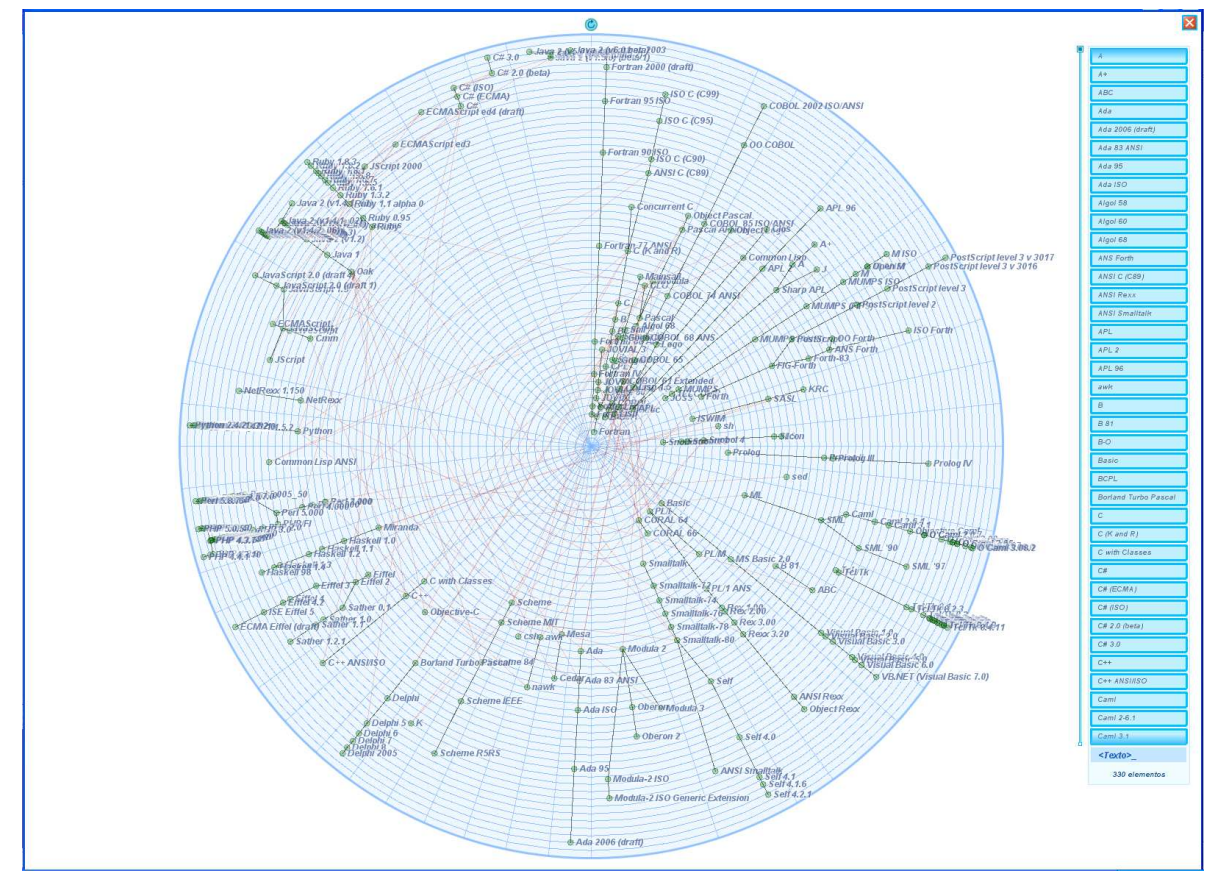

Fig. 5. Computer languages history, from 1957 to 2006, in Treevolution

Furthermore, the Levenez diagram suffers from the well-known problem of focus: as we scroll when we are following a particular time line, all contextual information is lost. Some tasks, common in the analysis of any hierarchicaltemporal data set, where the context is needed are:

- Have a clear overview of the whole evolution.

- Focus on a family or a subfamily while keeping its relationships with other families.

- Compare the evolution of two or more families.

- Identify a subfamily within a family.

- Focus on a particular period of time across families. 
The trade-off between global views and visibility of details is a long-standing problem in visualizations of large data sets [1]. Traditional distortion techniques can be used to focus on detail in particular areas while keeping the context. In addition to the visualization technique, for an effective data exploration, it is necessary to use some interaction and distortion techniques. Interaction techniques allow the data analyst to directly interact with the visualizations and dynamically change the visualizations according to the exploration objectives[14]. This approach has been followed in two ways in Treevolution:

- Sector distortion. The angle of any sector can be interactively opened in order to have more space for the layout of a particular subtree (or computer language family in this example). As one sector is opened, the remaining sectors are proportionally closed. This can be seen in Figure 6, where the PostScript sector (upper left quadrant) has been opened by the user in order to clearly see its hierarchy.

- Ring distortion. The width of any ring can be interactively increased in order to view more clearly that period of time. At this point, the tree-ring metaphor offers its best, since all rings cover the same amount of time, but the number of nodes within that period is variable (different growth index, in tree-ring chronology terms). This situation can be seen in Figure 7, where years 2002 and 2003 were very productive, with several versions. This way, although all rings represent a year time, the width of the rings has been increased in order to clearly view the rapid evolution of Java during that years. Note that inner years have their width decreased in order to make room for the productive years.

Furthermore, Treevolution can automatically weight the width of each family and the productivity of each year, so each sector and each ring is automatically distorted according to those weights. Thus, the resulting tree-ring layout can easily convey this complex information, that more traditional approaches cannot deal with (this is the case of the diagram in Figure 4, which is manually drawn by the author without using any automatic tool).

Besides, some more interactions [14] have been added in the Treevolution implementation. On the right hand size, an alphabetical browser of node labels permits to find a particular node within a complex hierarchy. Also, it is possible to filter the label browser with a word provided from the keyboard. This is what it was done in Figure 7: Oak was typed so the node was selected. As a result, the node is highlighted, its direct descendant is highlighted in blue, and its ancestors are highlighted in red. The oak sector (family) and the ring (year) are shadowed and the particular time is shown in the background (1991).

Figure 8 shows a similar situation but for the C language (Kernighan and Ritchie) in 1977. Note that a subfamily, C (K and R) is highlighted with a darker shadow than the whole family (Fortran). Also note that the Smalltalk family (in the bottom left quadrant) has a wider sector. This is the way Treevolution can maintain several foci while maintaining the context.

Another important feature of Treevolution is that the whole diagram can be rotated, in order to provide a way of avoiding the label cluttering that the 


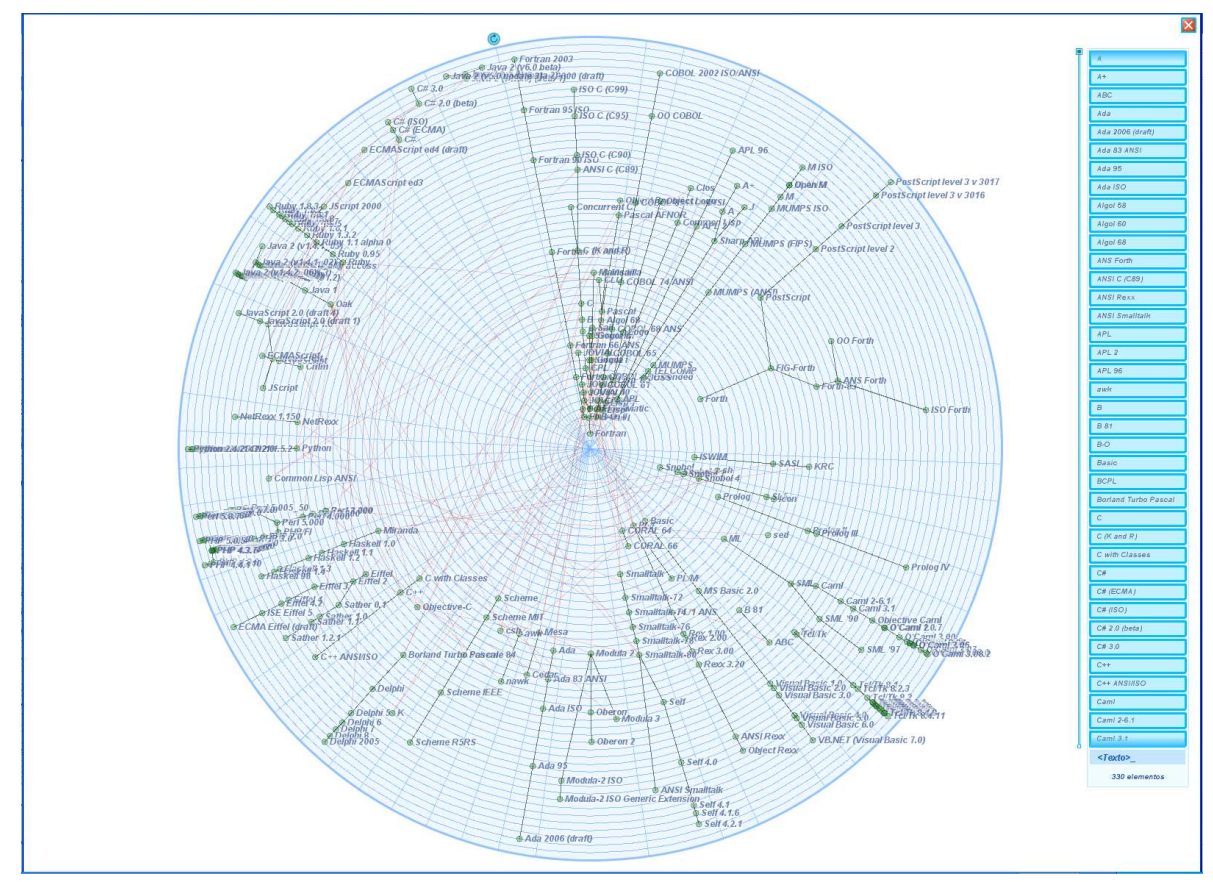

Fig. 6. The user is focused on the Postcript family (upper left quadrant)

automatic layout can produce. This a very important issue, since the design of an aesthetic label layout is an efficient way to increase the functionality (speeding up search tasks) of technical and scientific illustration [15]. The user only needs to drag the small round button that is located outside the circle. Note the different locations of this button in figures 5 (default location), 6,7 and 8 .

Finally, it should be noted that the visual engagement evoked by the natural tree is also due to its irregularities. These irregularities have not been taken into account for the current implementation of Treevolution. However, the variations in the ring width for different radios (differential growing) might be useful to display subtree features, and the variations in the colors (from heartwood to outer wood) might be useful to bring more clearly to mind the tree-ring outwardgrowing metaphor). This will be subject of further research.

\section{Conclusions}

A novel method for the visualization and navigation of hierarchical-temporal data was presented. The tree-ring metaphor provides an elegant solution to this particular problem, while the focus + context and interaction techniques permit the expert to see and understand a big and complex dataset at once. The rotation of the tree-ring is a simple but very efficient way of label uncluttering. As case 


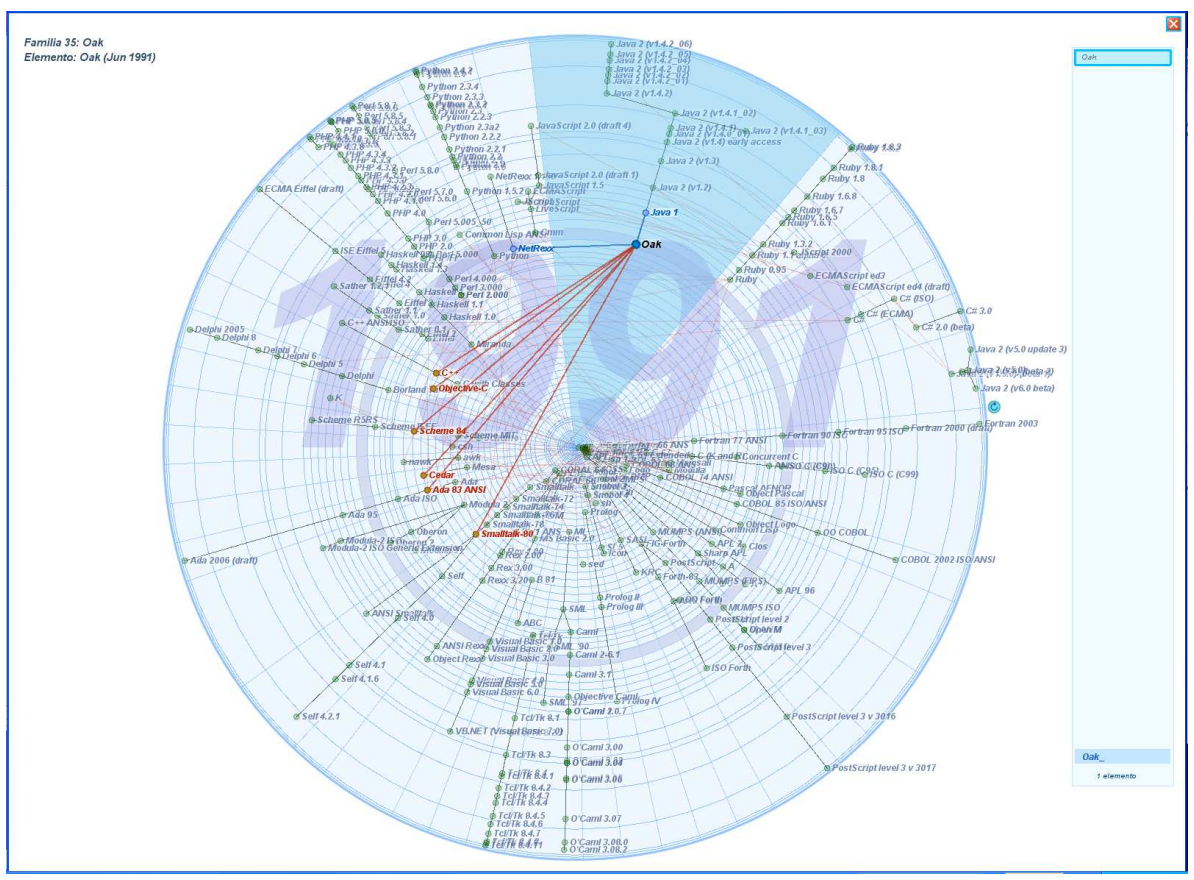

Fig. 7. Full details of the evolution of the Java hierarchy

study, Treevolution was successfully applied to the popular history of computer languages diagram, showing its potential for exploring hierarchies and helping the user to gain a deeper understanding of the evolution and relationships among the different elements in the hierarchy.

Future extensions will be providing multiple linked views of selected subtrees, fisheye view for rings, node filtering depending on a period of time, and test the technique with larger datasets. Also, the optimization of the layout algorithm, so the number of crossing edges can be minimized, will be studied.

Another important aspect that will be tested is the user performance (for instance, how the user judgments are affected by the orientation and order of the particular sequence around the circle).

Finally, a challenging issue is how to deal with trees whose structure changes over time, rather than just adding more nodes.

\section{References}

1. Herman, Melançon, G., Marshall, M.S.: Graph visualization and navigation in information visualization: A survey. IEEE Transactions on Visualization and Computer Graphics 6 (2000) 24-43

2. Battista, G.D., Eades, P., Tamassia, R., Tollis, I.G.: Graph Drawing: Algorithms for the Visualization of Graphs. Prentince Hall, Upper Saddle River, N. J. (1999) 
3. Shneiderman, B.: The eyes have it: A task by data type taxonomy for information visualizations. In: IEEE Visual Languages. Number UMCP-CSD CS-TR-3665, College Park, Maryland 20742, U.S.A. (1996) 336-343

4. Munzner, T., Burchard, P.: Visualizing the structure of the world wide web in $3 \mathrm{~d}$ hyperbolic space. In: VRML '95: Proceedings of the first symposium on Virtual reality modeling language, New York, NY, USA, ACM Press (1995) 33-38

5. Yee, K.P., Fisher, D., Dhamija, R., Hearst, M.A.: Animated exploration of dynamic graphs with radial layout. In: INFOVIS. (2001) 43-50

6. Eades, P.: Drawing free trees. Bulletin of the Institute for Combinatorics and its Applications 5 (1992) 10-36

7. Heer, J., Card, S.K., Landay, J.A.: prefuse: a toolkit for interactive information visualization. In: Proceedings of SIGCHI Human Factors in Computing Systems, New York, NY, USA, ACM Press (2005) 421-430

8. Lok, S., Feiner, S.: A survey of automated layout techniques for information presentations. In: Proceedings of SmartGraphics 2001. (2001)

9. Munzner, T., Guimbretière, F., Tasiran, S., Zhang, L., Zhou, Y.: Treejuxtaposer: scalable tree comparison using focus+context with guaranteed visibility. ACM Transactions on Graphics 22 (2003) 453-462

10. Morris, S.A., Yen, G., Wu, Z., Asnake, B.: Time line visualization of research fronts. Journal of the American Society for Information Science and Technology 54 (2003) 413-422

11. Robbins, K.A., Jeffery, C.L., Robbins, S.: Visualization of splitting and merging processes. Journal of Visual Languages and Computing 11 (2000) 593-614

12. Fry, B.: Computational Information Design. PhD thesis, MIT (2004)

13. National Visualization and Analytics Center: Illuminating the Path: The Research and Development Agenda for Visual Analytics. IEEE Press (2005)

14. Keim, D.A.: Information visualization and visual data mining. IEEE Transactions on Visualization and Computer Graphics 8 (2002) 1-8

15. Hartmann, K., Götzelmann, T., Ali, K., Strothotte, T.: Metrics for functional and aesthetic label layouts. Lecture Notes in Computer Sceinces, Smart Graphics: 5th International Symposium 3638 (2005) 115-127 


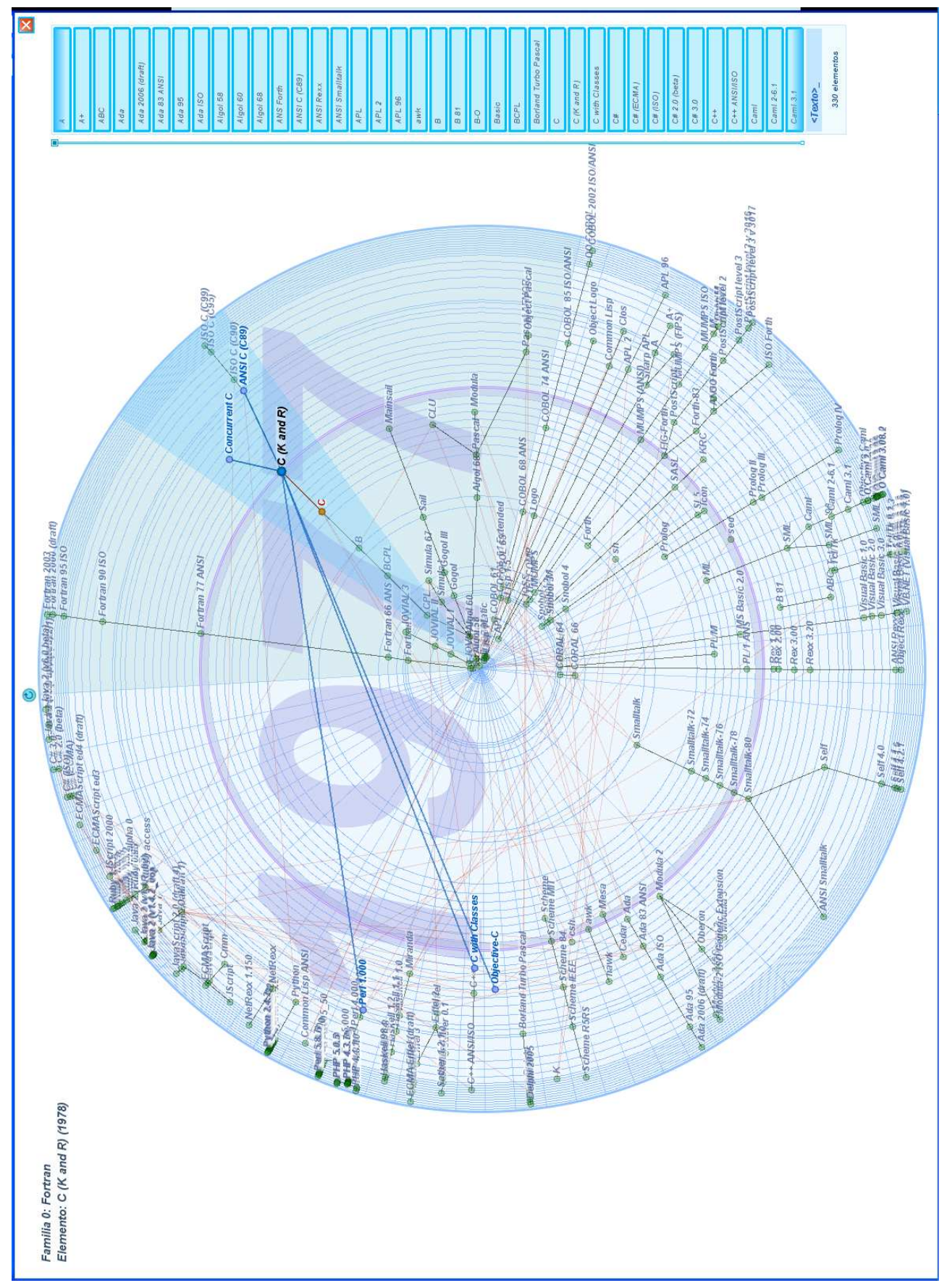

Fig. 8. Treevolution focusing on 1977 (Kernighan and Ritchie C family) 